Original Article

\title{
DOCKING STUDY OF METHYL HESPERIDIN AS NUCLEOSIDE REVERSE TRANSCRIPTASE INHIBITOR
}

\author{
HAFRIZAL RIZA ${ }^{\mathrm{a}^{*}}$, ANDHI FAHRURROJI ${ }^{\mathrm{a}}$, ARIF WICAKSONO ${ }^{\mathrm{a}}$, AHMAD KHARIS NUGROHO ${ }^{\mathrm{b}}$, SUDIBYO MARTONO $^{\mathrm{b}}$ \\ aDepartment of Pharmaceutics, Tanjungpura University, Pontianak, Indonesia, bepartment of Pharmaceutics, Gajah Mada University, \\ Yogyakarta, Indonesia \\ Email: hafrizal.riza@pharm.untan.ac.id \\ Received: 22 Sep 2017 Revised and Accepted: 22 Jan 2018
}

ABSTRACT

Objective: This study aims to analyze the methyl hesperidin physicochemical properties related to solubility and permeability, and the affinity of methyl hesperidin against reverse transcriptase HIV-1 activity as a competitive substrate.

Methods: This research was conducted using the computerized method, ChemOffice 15.0, to predict ligand physicochemical properties related to solubility and permeability, and Autodock Vina with Autodock Tools program to analyze ligand-receptor affinity.

Results: The analysis result of physicochemical properties of hesperidin and methyl hesperidin is respectively 300,27 g/Mol, 1,78, and 314,29 $\mathrm{g} / \mathrm{Mol}, 2,04$. The docking result shows that the binding energy of hesperidin, methyl hesperidin and zidovudine with receptor are respectively-8,0,8,8 and-9,3 kcal/Mol. The type of interactions between receptor and hesperidin is van der Waals and phi-phi staked, methyl hesperidin are van der Waals, hydrogen bond, phi-sigma, and phi-phi stacked, and zidovudine is an attractive charge, hydrogen bond, and phi-sigma.

Conclusion: Methyl hesperidin has good solubility and permeability, and has affinity with the receptor, a substrate of reverse transcriptase HIV-1.

Keywords; Methyl hesperidin, nucleoside reverse transcriptase inhibitor, Autodock Vina

(C) 2018 The Authors. Published by Innovare Academic Sciences Pvt Ltd. This is an open access article under the CC BY license (http://creativecommons.org/licenses/by/4.0/) DOI: http://dx.doi.org/10.22159/ijpps.2018v10i3.22724

\section{INTRODUCTION}

Acquired immunodeficiency syndrome (AIDS) is a global and deadly disease in the world. Based on the global report of the World Health Organization (WHO), the amount of death in the world caused by AIDS reached 1,8 million people [9]. Based on the data from the Health Ministry of Republic Indonesia, the incident number of AIDS in September 2014 reached 55.799 people in Indonesia [8]. Drug therapy for AIDS known as Retroviral Drug now has resistance and side effect problems affecting medication $[10,16,21]$. Therefore, there is a necessary to discover a new agent as anti-HIV. Nature is a source for anti-HIV because the drug discovery is begun from a plant [11].

Orange plant, one of a typical Kalimantan Barat plant, has a potential to be developed into antiHIV because this plant produces a few secondary metabolites such as hesperidin, tangeritin, hesperitin, naringin and naringenin. Hesperidin has been proved as antidiabetic, anticholesterol, antiinflammation, antioxidant and antifertility [6]. There are no toxic effects found in the trial of male and female mice. Moreover, hesperidin could be easily digested and is not accumulated in the body and hesperidin also does not cause an allergic reaction [5]. In another trial, 70\% ethanol extract of Justicia gendarussa Burm. $f$ leaves had been proved to inhibit the activity of reverse transcriptase HIV-1 and apigenin is the main secondary metabolite detected in the extract [4]. Apigenin had also been proved to inhibit the activity of reverse transcriptase HIV-1 as a competitive substrate, in vitro [3]. Hesperidin has a similar chemical structure with apigenin. They are flavonoid that has three rings with same chemical structures. Based on these data, hesperidin could possibly inhibit the activity of reverse transcriptase HIV-1.

Reverse transcriptase has an important role in the HIV life cycle because of the enzyme reversely transcript ribonucleic acid (RNA) virus into deoxyribonucleic acid (DNA) virus. DNA of virus next is incorporated into the DNA of the host cell and when host cell transcripts the code area containing this DNA, proteins needed to create a new virus are produced. All of those proteins and enzymes needed to create a new virus are originated from the host cell. Only reverse transcriptase, integrase and virus RNA are originally brought by the virus [7]. Therefore, the inhibition of the activity of reverse transcriptase HIV-1 could stop virus replication.

Although hesperidin has a potency as an anti-HIV activity, hesperidin could not be absorbed well in the body. Solubility, one of the physicochemical properties of hesperidin is low in the body causing low bioavailability. The solubility in water, stomach and intestine are respectively $57 \mathrm{mg} / \mathrm{l}, 49,1 \mathrm{mg} / \mathrm{l}$ and 68,0 mg/l [2]. Log P of hesperidin are 0,30 [1]. Therefore, there is a necessary to improve the solubility of hesperidin. Chemical structure modification is a way to improve hesperidin solubility by methyl addition, a nonpolar functional group, into hesperidin chemical structure and also eliminates the glicon functional group from the structure.

It is needed to analyze that the addition of methyl into the chemical structure does not change the anti-HIV activity of hesperidin. The one of research methods could be chosen is a computerized method. The method could predict the solubility and the affinity of interaction between ligand and receptor. This study aims to analyze the physicochemical properties related to hesperidin solubility and permeability properties in the body and the docking study of methylated hesperidin as a competitive substrate of reverse transcriptase HIV-1.

\section{MATERIALS AND METHODS}

\section{Materials}

Hardware used for physicochemical property analysis and docking study of ligand is Notebook PC ASUS X455L Series with the specifications of Intel Core i3-4030U CPU 1,90 GHz, system operation Windows 8,1 Enterprise with 64-bit windows and 2,00 GB of RAM. Software used for physicochemical property analysis of ligand is ChemDraw 15.0. Software used for docking study of ligand is Autodock Vina with Autodock Tools 1.1.2 version and software used for docking visualization of ligandreceptor interaction is Discovery Studio.

\section{Docking methodology}

2 dimensions (2D) and 3 (3D) dimensions, the chemical structure of ligand was obtained by drawing ligand structure and setting the 
minimal sterical energy of ligand structure. Receptor, reverse transcriptase HIV-1 substrate, was downloaded from RCSB Protein Data Bank (http://www.rcsb.org/pdb/home/home.do). Grid parameter was set using Autodock Tools to coordinate-18.177 x 25.99 x $37.741 \mathrm{~A}$ (Amstrong) and dimension 30 x 30 × $30 \mathrm{~A}$. Grid dimension was set same between the ligand and positive control. Docking study was calculated using Autodock Vina with the grid parameter and type of interaction was visualized using Discovery Studio.

\section{The analysis of physicochemical properties}

Physicochemical properties of ligand were analyzed using Chem Draw 15.0 [12].

\section{Data analysis}

The analysis result of ligand physicochemical properties (LogP, BM, $\mathrm{H} \mathrm{H}$ acceptor and donor) are analyzed using the rule of five Lipinski to predict the properties of ligand absorption and permeability. The affinity of ligand-receptor interaction was analyzed using Autodock Vina and Discovery Studio to analyze ligand-receptor binding energy and amino acid residues involved from ligand-receptor interaction (kcal/Mol) [18-20]

\section{RESULTS AND DISCUSSION}

Chemical structure of modification is a part of drug development. Addition of functional group of atoms in the chemical structure of a compound would change the polarity and affinity of a compound to a receptor [12]. In order to certain, the effect of methyl addition into hesperidin against the solubility and the affinity of methyl hesperidin to the receptor, physicochemical property analysis and docking study was conducted in this research based on a very large mathematical operation in computational chemistry. The method integrates chemical theory and modelling with experimental observations based on mathematical algorithms, statistics and databases. It calculates the fundamental laws of physic of an atom such as charged particles, positive nuclei, negative electrons and geometrical arrangements of the nuclei correspond to stable molecules, and how atoms interact each other. Therefore, this method could contribute as preliminary studies for supporting in vitro and in vivo experimental method [18].

3D chemical structures of hesperidin, methyl hesperidin and (positive control) zidovudine obtained using ChemDraw and after minimizing energy are shown in fig. 1.

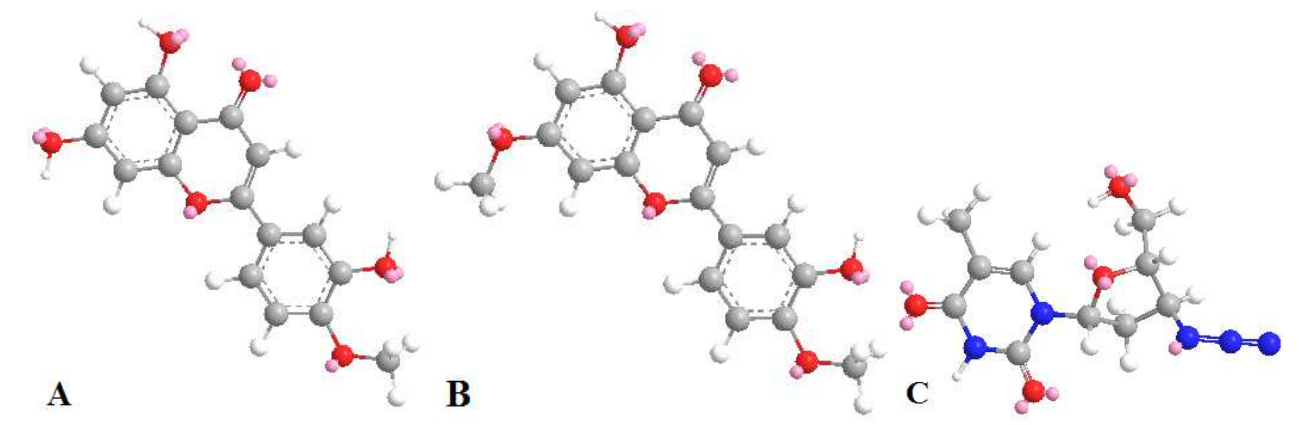

Fig. 1: Ligand 3D structure obtained using ChemDraw 15.0, A): Hesperidin, B): methyl hesperidin, C): zidovudine

Minimizing the energy of ligand structure obtained using Chem Office program is conducted to obtain the most stable conformation of the ligand that are close to the real ligand binding to the receptor in the human body. In order to obtain the confirmation of a compound that is as similar as the one in the nature, the minimizing energy would give the best arrangement of atoms position in a space for a compound so net inter-atomic force and sterical energy of each atom are close to zero and the position on the potential energy surface is a stationary point. The confirmation of structure would affect the real physicochemical properties of a compound. It also affects the docking score showing the affinity of ligand-receptor [13].

The analysis result of ligand physicochemical properties showed that $\log \mathrm{P}$ of methyl hesperidin is more than hesperidin. Same as hesperidin, methyl hesperidin have a molecular weight less than 500 and $\mathrm{C} \log \mathrm{P}$ is less than 5 . The amount of $\mathrm{N}-\mathrm{H}$ functional group for Amida are 0 , and $\mathrm{O}-\mathrm{H}$ functional group for carboxylate and phenol are 2 , the total are 2, means less than 5 . The amount of 0 atom is 5 and $\mathrm{N}$ atom are 0 , so the total are 5 , means less than 10 . According to Lapinski Rule, these could be predicted that hesperidin and methyl hesperidin could be absorbed and has good permeability.

Lapinski Rule reveals that a drug would be pharmacology active if it has physicochemical properties contributing to drug pharmacokinetics in the human body including absorption, distribution, metabolism and excretion. This rule proved based on empirically screening program for the most oral drug that a range of physical properties is consistent with oral activity such as Acyclovir, Alprazolam, Proxicam, Vinblastin and 2000 others. In general, a drug has low absorption and permeability because the molecular weight is more than 500 Dalton, an octanol-water partition of coefficient log $P$ is greater than 5, hydrogen bond acceptors of a drug are more than 10 for nitrogen or oxygen atoms, and hydrogen bond donors for a drug are more than 5 for nitrogen or oxygen atoms with one or more hydrogen atoms [12].
Both ligand, methyl hesperidin, hesperidin and positive control were optimized using Autodock Tools to determine various factors contributing to ligand affinity to receptors such as partial charges, the addition of both polar and nonpolar hydrogen atoms, rotatable bonds, the number of torsional degrees, identifying an atom as a central root and the torsion count.

Crystal structure of the receptor was downloaded from RCSB Protein Data Bank (http://www.rcsb.org/pdb/home/home.do), in the form of reverse transcriptase HIV-1 complex with DNA and zidovudine, with the code of receptor 3V4I. The receptor was optimized using Autodock Tools such as eliminating water, adding hydrogen atoms and partial charges.

Grid parameters for methyl hesperidin and hesperidin were set close to positive control grid box by choosing the similar dimension area. It was conducted in order to keep the docking result is in the active site of the receptor. The active site of receptor chosen are amino acid residues affecting the function or activity of the enzyme.

The binding energy score for methyl hesperidin are different 0.8 $\mathrm{kcal} / \mathrm{mole}$ than hesperidin binding energy score as shown in table 1 . The binding energy score of hesperidin and methyl hesperidin, and positive control, zidovudine are lower than zero. This means that the binding of ligand and receptor cause releasing energy called exoterm. Binding energy shows the inter-atomic force, the sterical energy and potential energy resulted from ligand-receptor binding. The lower energy binding score from ligand-receptor binding, the more stable the ligand-receptor to bind. This because the better affinity of ligand-receptor is determined by the stability of ligandreceptor binding[13]. The RMSD score shows the iteration of conformation model of ligand that could bind to receptor with the binding energy score shown in table 1 is significantly not different [15]. It could be predicted that methyl hesperidin, hesperidin could be bound to the receptor as positive control. 
Table 1: Binding energy and binding site obtained using autodock vina 1.1.2.

\begin{tabular}{lll}
\hline Ligand & Binding energy score (kcal/Mol) & RMSD \\
\hline Hesperidin & -8.0 & 0,000 \\
Methyl Hesperidin & -8.8 & 0,000 \\
Zidovudine & $-9,3$ & 0,000 \\
\hline
\end{tabular}

Hesperidin, methyl hesperidin and Zidovudin could inhibit the activity of the enzyme, reverse transcriptase. This could be proved first by comparing the hesperidin and methyl hesperidin binding energy score with a proven antiretroviral drug, Zidovudin. Second, the relationship between binding energy score with the bioactivity of the drug is shown in a docking study of Glimepirid and Glibenclamid, antidiabetic drugs with the binding energy score that is lower than zero [19]. Moreover, another study shows the relationship between the binding energy score with bioactivity study. A compound in Buddleja salviifolia (L). Lam leaf showed the binding energy score is lower than zero and has bioactivity as anti-inflammatory and antioxidant [20].

Type of interaction between ligand, hesperidin and receptor, DNA, using Discovery Studio is shown in fig. 2.

Type of interaction between ligand, methyl hesperidin and receptor, DNA, using Discovery Studio is shown in fig. 3.

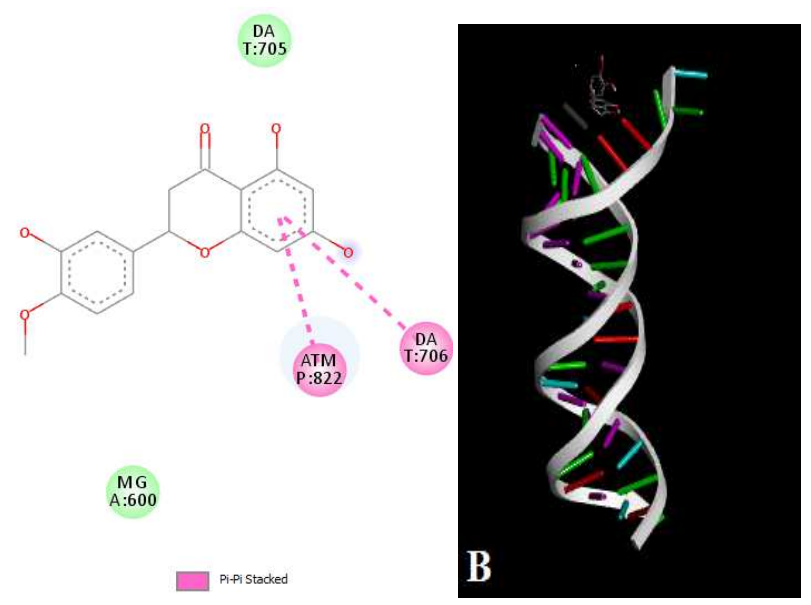

Fig. 2: Visualization of hesperidin-receptor interaction using discovery studio, A): Type of interaction between hesperidin and receptor, B): Hesperidin is in the position of the receptor substrate

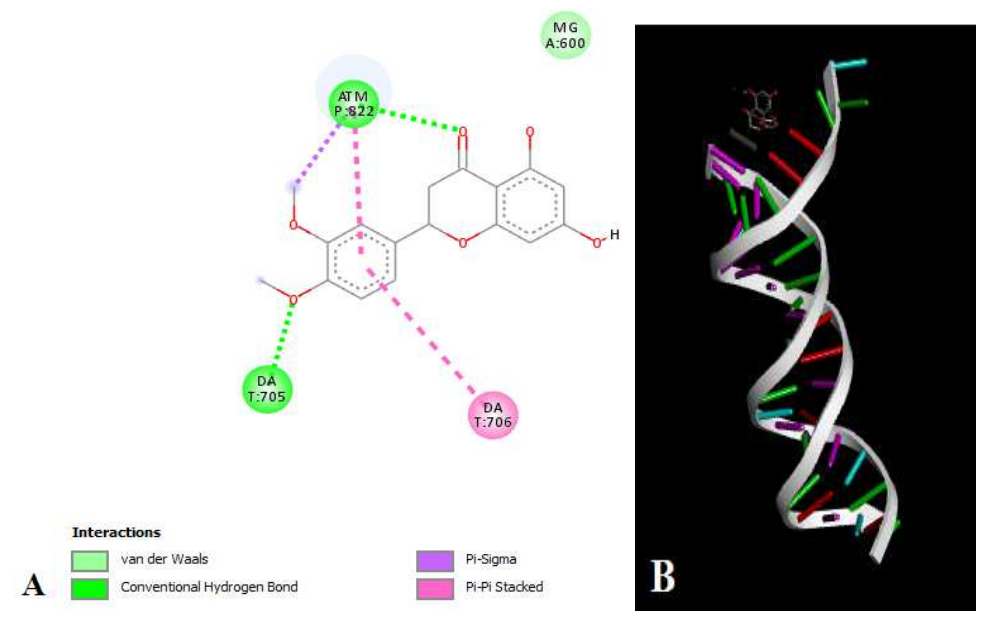

Fig. 3: Visualization of methyl hesperidin-receptor interaction using discovery studio, A): Type of interaction between methyl hesperidin and receptor, $B$ ): Methyl hesperidin is in the position of the receptor substrate

Type of interaction between methyl hesperidin and receptor is more than hesperidin and the receptor as shown in fig. 2 and 3 . This contributes to the different binding energy score between both ligands. Both ligands could be bound in the active site of the substrate position of receptor shown in fig. 2 and 3. Because both ligands have no atoms that could facilitate phosphate bond to the next incoming substrate, both ligands could act as stop codon for reverse transcription of virus RNA into DNA [14].
Type of interaction between positive control, zidovudine, and DNA using Discovery Studio are shown in fig. 4. Zidovudine has the most interactions contributing to the greatest zidovudine binding energy score. Zidovudine could be bound in the substrate position of the receptor. Because zidovudine has no atoms that could facilitate phosphate bond to the next incoming substrate, zidovudine could act as stop codon for reverse transcription of virus RNA into DNA [14]. 


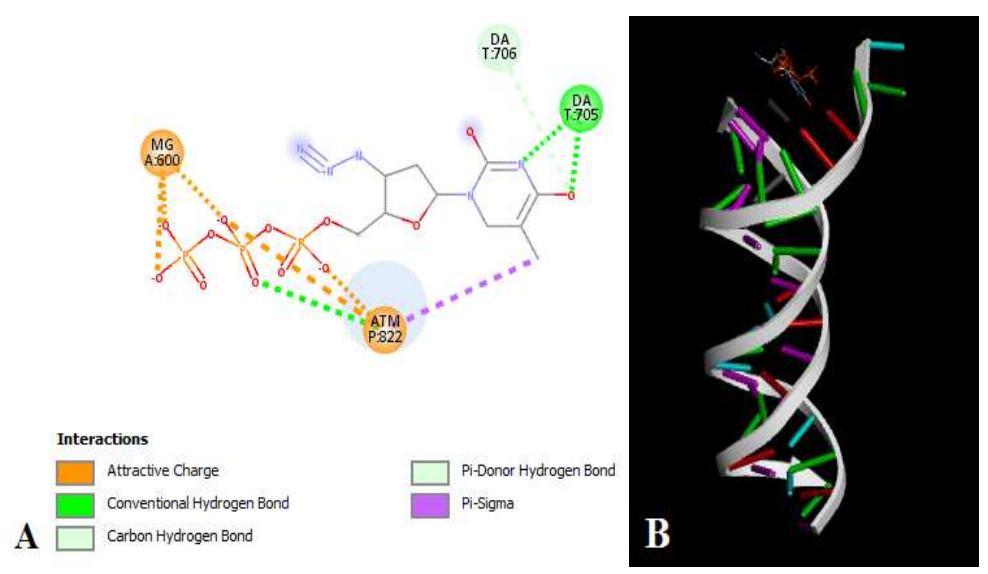

Fig. 4: Visualization of zidovudine-receptor interaction using discovery studio, A): Type of interaction between zidovudine and receptor, B): Zidovudine is in the position of the receptor substrate

Hesperidin and methyl hesperidin could inhibit the activity of reverse transcriptase because of both ligand bind to an amino acid residue in the active site of the substrate. There is a relationship between the binding sites of docking studies with the bioactivity of the drug. In a study of proven antidiabetic drugs showed that Glimepiride bind to the different kind of amino acid residue with other antidiabetic drug but both ligand has good bioactivity [19]. In other studies showed that there is a relationship between docking studies and the bioactivity of the compound in Buddleja salviifolia (L). Lam leaf \{20], and the consistent result between docking result and in vitro antibacterial of $\mathrm{N}$-[(2-chloroquinolin-3-yl) methylidene $]$ 2 -aniline [17]. This relationship could be obtained because the different kind of amino acid residue is located in the active site of receptor whose receptor activity.

In order to obtain the information about the methyl hesperidin activity as anti-HIV, especially related to the quantity of compound that could inhibit the activity of reverse transcriptase, we need to conduct a research experiment. Computational chemistry is a qualitative method used to determine whether or not atoms of a ligand interaction with atoms of a receptor. The method was conducted only based on the calculation of theoretical approach [18].

\section{CONCLUSION}

The results first showed that according to Lapinski Rule, methyl hesperidin has physicochemical properties contributing to drug pharmacokinetics in the human body. It could be predicted that methyl hesperidin would be pharmacology active. The second showed that the binding energy of methyl hesperidin is lower than zero, as zidovudine, a proven antiretroviral drug. It means that methyl hesperidin could be bound to reverse transcriptase as competitive substrate. Therefore, It could be predicted that methyl hesperidin could be absorbed and penetrated into the human body, and could inhibit the activity of reverse transcriptase HIV-1.

\section{ACKNOWLEDGEMENT}

We would like to express gratitude to the Ministry of Research and Higher Education and Faculty of Medicine of Tanjungpura University for their support in research funding and facilities, also Faculty of Pharmacy of Gadjah Mada University for the cooperation in conducting supervision.

\section{AUTHORS CONTRIBUTIONS}

Hafrizal Riza participated in the physicochemical properties and docking analysis, and the data interpretations, Andhi Fahrurroji and Arif Wicaksono participated in the writing the manuscript, Ahmad Kharis Nugroho and Sudibyo Martono participated in the review of research proposed.

\section{CONFLICT OF INTERESTS}

Declared none

\section{REFERENCES}

1. Oliveira RMM, Daniel JFS, Aguiar I, Silva MFGF, Fernandes JB, Carlos RM. Structural effects on the hesperidin properties obtained by chelation to magnesium complexes. J Inorg Biochem 2013;129:35-42.

2. Sansone F, Alessandra R, Pasquale DG, Francesco DS, Rita PA, Maria RL. Hesperidin gastroresistent microparticles by spraydrying: preparation, characterization, and dissolution profiles. AAPS PharmSciTech 2009;10:391-401.

3. Prajogo B, Widiyanti P, Riza H. Effect of ethanolic extract of Justicia gendarussa Burm $\mathrm{f}$ against the activity of reverse transcriptase HIV enzyme in vitro. J Bahan Alam Indonesia 2014;8:384-8.

4. Prajogo B, Dudi S, Mulya HS. Analisis kadar gendarusin a pada tanaman budidaya Justicia gendarussa burm. f. J Farmasi Indonesia 2007;3:176-80.

5. Wilsen PK, Spada DS, Salvador M. Antioxidant activity of flavonoid hesperidin in chemical and biological systems. J Agric Food Chem 2005;53:4757-61.

6. Garg A, Garg S, Zaneveld LJD, Singla AK. Chemistry and pharmacology of the citrus bioflavonoid hesperidin. Phytother Res 2001;15:655-69.

7. Pommier Y, Johnson AA, Marchand C. Integrase inhibitors to treat HIV/AIDS. Nat Rev Drug Discovery 2005;4:236-48.

8. Kementerian Kesehatan Republik Indonesia, Laporan Situasi Perkembangan HIV and AIDS di Indonesia sampai dengan Juni; 2011. Available from: http://www.aidsindonesia.or.id/ download/lT2Menkes2011.pdf. [Last accessed on 13 Nov 2011]

9. World Health Organization, Global Summary of the HIV AIDS epidemic; 2009. Available from: http://www.who.int/hiv/ data/2009_global_summary.png [Last accessed on 10 Nov 2011]

10. Flexner C. HIV Protease Inhibitor. N Engl J Med 1998;338:1281-93.

11. Feher M, Schmidt JM. Property distributions: differences between drugs, natural products, and molecules from combinatorial chemistry. J Chem Inf Comput Sci 2003;43:218-27.

12. Block JH. Drug design strategies. In: Beale JM, Block JH. Eds. Wilson and Gisvold's Textbook of Organic Medicinal and Pharmaceutical Chemistry. 12th Ed. Philadelphia: Lippincott Williams and Wilkins; 2011. p. 38-9.

13. Jensen F. Introduction to computational chemistry. 2nd Ed. Hoboken: United States: John Wiley and Sons Ltd; 2006. p. 69416.

14. Brian D. Herman, Nicolas Sluis-Cremer. Molecular Pharmacology of Nucleoside and Nucleotide HIV-1 Reverse Transcriptase Inhibitors, Pharmacology. Dr. Luca Gallelli Ed. Pittsburgh: Intech; 2012. p. 63-80.

15. Alberto C, Mattia S, Ivana M, Antonella C, Stefano M. Dock bench: an integrated informatic platform bridging the gap between the robust validation of docking protocols and virtual screening simulations. Molecules 2015;20:9977-93. 
16. Rajesh CS. Comparative study of the effect of Swertia chirata leaf extract on Indinavir treated rats. Asian J Pharm Clin Res 2017;10:339-42.

17. Pradeep PS, Shrungesh Kumar TO, Prashantha N, Mahadevan KM. Synthesis, in vitro antibacterial, toxicity and molecular docking anticancer activity of novel N-[(2-chloroquinolin-3-yl) methylidene]-2-aniline Schiff bases. Int J Curr Pharm Res 2015; 7:37-46.

18. Jensen F. Introduction to Computational Chemistry. 2nd Ed. Denmark: John Wiley and Sons, Ltd; 2007. p. 1-21.
19. Ruiter JD. Overview of antidiabetic the agents. Endocrine Pharmacotherapy Module. Spring; 2013. p. 3-29.

20. Pendota SC, Ndhala AR, Aremu AO, Aderogba MA, Van Staden J. Anti-inflammatory, antioxidant and in silico studies of Buddleja salviifolia (L). Lam leaf constituents. S Afr J Bot 2014;93:79-85.

21. Mallya SD, Kamath VG, Nair S, Kamath A. Does providing free antiretroviral therapy ensure optimal adherence among people living with human immunodeficiency virus/acquired immunedeficiency syndrome. Asian J Pharm Clin Res 2016;19:1-4. 